\title{
Relationship between Applied Static Magnetic Field Strength and Thermal Conductivity Values of Molten Materials Measured Using the EML Technique
}

\author{
Yuya BABA ${ }^{1}$, Ken-ichi SugioKA ${ }^{1}$, Masaki KuBO ${ }^{1}$, \\ Takao TsuKAdA ${ }^{1}$, Kazutoshi Sugie ${ }^{2}$, Hidekazu KobataKe ${ }^{2}$ \\ and Hiroyuki FUKUYAMA ${ }^{2}$ \\ ${ }^{1}$ Department of Chemical Engineering, Tohoku University, \\ 6-6-07, Aramaki, Aoba-ku, Sendai-shi, Miyagi 980-8579, Japan \\ ${ }^{2}$ Institute of Multidisciplinary Research for Advanced Materials, \\ Tohoku University, \\ 2-1-1, Katahira, Aoba-ku, Sendai-shi, Miyagi 980-8577, Japan
}

Keywords: Thermal Conductivity, Molten Materials, EML Technique, Static Magnetic Field

\begin{abstract}
In order to quantitatively investigate the effect of convection in an electromagnetically levitated molten iron droplet on the thermal conductivity of the droplet measured by the electromagnetic levitation (EML) technique in the presence of a static magnetic field, numerical simulations of the melt convection in the droplet and measurements of the thermal conductivity with the periodic laser heating method were carried out. In addition, the thermal conductivity of molten iron was measured by the EML technique, and then compared with values obtained numerically. It was found that the numerical simulations could sufficiently explain the measurement of thermal conductivity by the EML technique in a static magnetic field. It is suggested that a static magnetic field of $10 \mathrm{~T}$ is enough to measure the real thermal conductivity of molten iron by the EML technique. Moreover, the correlation between the static magnetic field and the contribution of melt convection to the measured thermal conductivity was investigated by using a nondimensional parameter, which is the ratio of the electromagnetic force operated by the static magnetic field to that produced by the alternating current in the RF coils.
\end{abstract}

\section{Introduction}

In materials processing at high temperatures, e.g., silicon single crystal growth, precision casting of superalloy turbine blades, or precision joining, it has been well recognized that numerical simulation is an effective tool for the design and optimization of processes. Since thermophysical properties are indispensable as input data in numerical simulations, it is important to establish a method to precisely measure the thermophysical properties of high-temperature molten materials and to collect and systematize data on the thermophysical properties. The electromagnetic levitation (EML) technique has recently been used for measuring the thermophysical properties of molten materials (Egry et al., 2001). In this technique, an alternating electric current in RF coils induces an eddy current in a conductive material sample, and the sample is melted by Joule heating resulting from the current. In addition, the electromagnetic force generated by the interaction between an alternating magnetic field and the induced current lifts up the molten dropshaped material. Therefore, the containerless EML tech-

Received on February 9, 2011; accepted on March 23, 2011

Correspondence concerning this article should be addressed to

K. Sugioka (E-mail address: ken_sugioka@pcel.che.tohoku.ac.jp). nique facilitates the precise measurement of the thermophysical properties of highly reactive molten materials, such as viscosity, density, and surface tension, over a wide temperature range even under an undercooled condition in the absence of contamination.

However, it might be difficult to measure the thermal conductivity of molten materials by using the technique because magnetohydrodynamic (MHD) convection due to the electromagnetic force occurs in a molten droplet, together with buoyancy and Marangoni convection, and the flow velocity reaches $10-40 \mathrm{~cm} / \mathrm{s}$ (Zong et al., 1992; Li and Song, 1998; Bojarevics and Pericleous, 2003; Hyers, 2005). Obviously, such intensive melt convection affects the thermal field in the droplet and, consequently, the measured thermophysical properties. Therefore, the thermal conductivity measured in the presence of convection is an effective thermal conductivity and is affected by convective heat transfer in addition to conduction. To measure the thermal conductivity accurately, Kobatake et al. (2007, 2008, 2010a) and Fukuyama et al. (2009) have developed a novel method to measure the thermal conductivity of molten materials by the EML technique, where the measurement method is based on periodic laser heating and a static magnetic field is applied to suppress melt convection in the droplet. Using this method, they have measured the ther- 
mal conductivities of molten silicon (Kobatake et al., 2007, 2008, 2010a) and molten stainless steel (Fukuyama et al., 2009). In addition, Tsukada et al. (2009) and Sugioka et al. (2010) have carried out numerical simulations of melt convection in an electromagnetically levitated silicon droplet in a static magnetic field and compared the results with experimental results; they demonstrated that the EML technique together with the application of a static magnetic field is effective to precisely measure the thermal conductivity of molten silicon, and they proposed an optimum static magnetic field for the measurement of the real thermal conductivity of molten silicon. Using such an EML technique and by applying a static magnetic field, we plan to measure the thermal conductivities of a variety of molten materials whose conductivities have not been measured yet at high temperatures.

Recently, in a series of studies, we measured the thermal conductivity of molten iron by the EML technique (Sugie et al., 2010). Although iron is a primary component of steel materials, for instance, high-strength steel sheets produced by thin-slab casting, there is no available data on its thermal conductivity in the molten state in a wide temperature range, including the supercooled condition. Most of the data obtained in previous studies (Touloukian et al., 1970; Ostrovskii et al., 1980; Zinovyev et al., 1986; Mills et al., 1996; Nishi et al., 2003) pertained to thermal conductivity at the melting point temperature or in a relatively narrow range of temperatures above the melting point. In this study, we numerically and experimentally investigated the effect of a static magnetic field on the thermal conductivity of molten iron measured by the EML technique. We propose an optimum value of the static magnetic field to measure the real thermal conductivity of molten iron. In addition, by using the thermal conductivity of molten nickel and molten iron measured in this work, along with the thermal conductivity of molten silicon measured in previous studies (Kobatake et al., 2007, 2008, 2010a; Sugioka et al., 2010), the correlation between the static magnetic field strength and the measured values of thermal conductivity was investigated by considering nondimensional parameters.

\section{Numerical Simulation}

Figure 1 shows a schematic diagram of the EML system used to measure the thermal conductivity of molten iron. For the measurement of the thermal conductivity of molten nickel, an RF coil system with the same geometry as that shown in Figure 1 was used, but the position of the droplet relative to the RF coil was different. In the numerical simulation, the electromagnetic field in the system shown in the figure was first computed to obtain the distributions of the electromagnetic force and heat generation rate in an electromagnetically levitated droplet, and then the flow and temperature

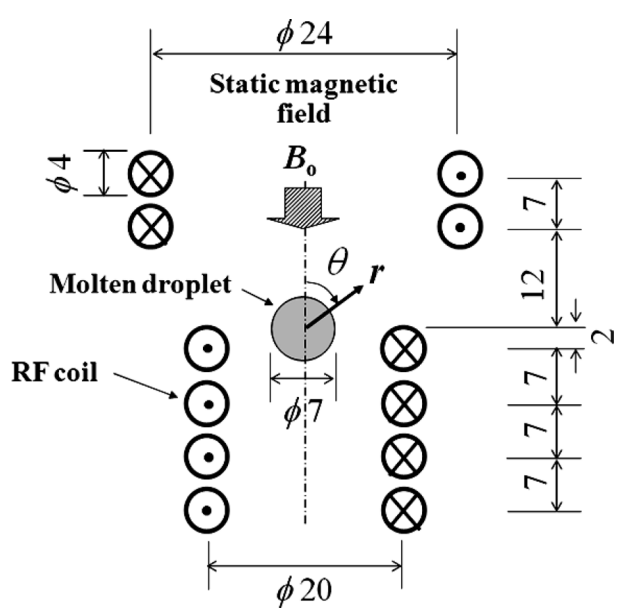

Fig. 1 Schematic diagram of an electromagnetic levitator used for measuring the thermal conductivity of molten iron

fields in the droplet in the presence of the alternating magnetic field generated by the RF coils and the static magnetic field applied to suppress melt convection were calculated. Moreover, the periodic laser heating used for measuring thermal conductivity was simulated numerically by considering the melt convection in the droplet, in order to investigate the effect of the static magnetic field on the measured thermal conductivity. For the convenience of the readers, an outline of our model is given below. The details are given in the paper of Tsukada et al. (2009).

In the numerical simulation of the electromagnetic field, the following assumptions were made: (1) the shape of an electromagnetically levitated droplet is spherical, (2) the system is axially symmetric, (3) the media are linear, isotropic and stationary, (4) the displacement current is negligible, (5) there is no net charge in the system, and (6) every physical quantity has conventional harmonic time dependence. Under these assumptions, the scalar equation for the azimuthal component of a vector potential derived from Maxwell's equation was solved under appropriate boundary conditions; the hybrid finite difference method (FDM) and boundary element method (BEM) were used to treat the field in unbounded free space.

In the numerical simulations of the flow and temperature fields in a droplet, we considered the MHD convection resulting from the distribution of the electromagnetic force generated by the alternating current in the RF coils, and the buoyancy and Marangoni convection in the droplet, which are suppressed by applying an axial static magnetic field $B_{0}$. The continuity, Navier-Stokes, and energy equations were solved under the following assumptions: (1) the droplet shape is spherical, (2) the system is axially symmetric, (3) the flow is laminar and the fluid is incompressible, and (4) the thermophysical properties of the melt are constant, except for the tempera- 
Table 1 Thermophysical properties of molten iron and nickel used in numerical simulations

\begin{tabular}{lcc}
\hline & $\mathrm{Fe}$ & $\mathrm{Ni}$ \\
\hline Temperature coefficient of surface tension $[\mathrm{N} /(\mathrm{m} \cdot \mathrm{K})]$ & $\left.4.18 \times 10^{-4} \mathrm{a}\right)$ & $\left.3.80 \times 10^{-4} \mathrm{a}\right)$ \\
Viscosity $[\mathrm{Pa} \cdot \mathrm{s}]$ & $\left.5.50 \times 10^{-3} \mathrm{a}\right)$ & $4.90 \times 10^{-3}$ a) \\
Density $\left[\mathrm{kg} / \mathrm{m}^{3}\right]$ & $7.00 \times 10^{3 \mathrm{~b})}$ & $\left.7.91 \times 10^{3} \mathrm{a}\right)$ \\
Volume expansion coefficient $[1 / \mathrm{K}]$ & $1.53 \times 10^{-4 \mathrm{~b})}$ & $\left.1.51 \times 10^{-4} \mathrm{a}\right)$ \\
Thermal conductivity $[\mathrm{W} /(\mathrm{m} \cdot \mathrm{K})]$ & $39.0^{\mathrm{c})}$ & $56.8^{\mathrm{d})}$ \\
Specific heat $[\mathrm{J} / \mathrm{kg}]$ & $807^{\mathrm{c})}$ & $620^{\mathrm{a})}$ \\
Emissivity $[-]$ & $0.4^{\mathrm{e}}$ & $0.364^{\mathrm{d})}$ \\
Melting point temperature $[\mathrm{K}]$ & $\left.1808^{\mathrm{a}}\right)$ & $\left.1727^{\mathrm{a}}\right)$ \\
Electric conductivity $[1 /(\Omega \cdot \mathrm{m})]$ & $7.00 \times 10^{5 \mathrm{a})}$ & $1.18 \times 10^{6}$ a) \\
\hline
\end{tabular}

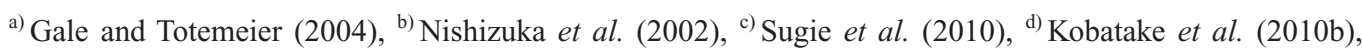

e) Kobatake (2010)

Table 2 Processing parameters used in the numerical simulations

\begin{tabular}{lcc}
\hline & $\mathrm{Fe}$ & $\mathrm{Ni}$ \\
\hline Frequency of electric current in RF coil $[\mathrm{kHz}]$ & 280 & 280 \\
Electric current in RF coil [A] & 520 & 520 \\
Diameter of cross area of RF coil [m] & $4.0 \times 10^{-3}$ & $4.0 \times 10^{-3}$ \\
Droplet diameter [m] & $7.0 \times 10^{-3}$ & $7.0 \times 10^{-3}$ \\
Heat transfer coefficient $\left[\mathrm{W} /\left(\mathrm{m}^{2} \cdot \mathrm{K}\right)\right]$ & $1.67 \times 10^{2}$ & $1.62 \times 10^{2}$ \\
Ambient temperature $[\mathrm{K}]$ & 293 & 293 \\
Static magnetic field $[\mathrm{T}]$ & $4-20$ & $3-10$ \\
\hline
\end{tabular}

ture dependence of the density and surface tension. The above-mentioned governing equations for the flow and temperature fields were solved numerically by employing the control-volume-based finite difference technique; the SIMPLER algorithm was used to obtain the velocity field by coupling the continuity and momentum equations.

Moreover, in order to numerically determine the thermal conductivity, the temperature responses at the lower part of the droplet were evaluated by solving the thermal advection-conduction equation for the droplet when the upper part was periodically heated by a modulated laser light source, similar to the experiment; the flow field in the droplet described above was fixed by assuming that the flow field is not affected by periodic heating because the predominant flow in the droplet is the MHD convection.

As mentioned in the next section, the temperature of the levitated droplet was controlled by blowing $\mathrm{He}$ gas on the droplet surface during the measurement. To consider such a cooling process for the droplet in the numerical simulations, both convective and radiative heat loss from the droplet surface to the ambient were added to the boundary condition for the thermal field at the droplet surface. In previous numerical simulations for molten silicon (Sugioka et al., 2010), the heat loss from the droplet surface was considered to be only radiative.
Tables 1 and $\mathbf{2}$ show the thermophysical properties and processing parameters, respectively, used in the numerical simulations here.

\section{Thermal Conductivity Measurement}

Using the electromagnetic levitator shown in Figure 1 , an iron sample with a purity of $99.5 \%$ and a mass of $1.3 \mathrm{~g}$ was heated, melted, and levitated, and the thermal conductivity of the molten iron was then measured by the periodic laser heating method. The details of the measurement procedure are given in the paper of Sugie et al. (2010). In the periodic laser heating method, the upper part of an electromagnetically levitated droplet is periodically heated by a modulated light source (a semiconductor laser is used in this work) and the temperature variation at the lower part of the droplet is detected using a pyrometer; the spot radius used in this study was $1.5 \mathrm{~mm}$. In this work, the $e^{-2}$ radius of the laser beam was $2.0 \mathrm{~mm}$. The amplitude of the laser power was $13.8 \mathrm{~W}$, and the frequencies of the laser power $\omega$ were varied between 0.04 and $0.3 \mathrm{~Hz}$. The RF coil shown in Figure 1 was installed inside a superconducting magnet, where the frequency of the RF current was $280 \mathrm{kHz}$, and the static magnetic field applied to suppress convection in the droplet was 3 to $10 \mathrm{~T}$. All the measurements were carried out in an $\mathrm{He} / \mathrm{Ar}+5 \% \mathrm{H}_{2}$ atmosphere and at 
$1767-2085 \mathrm{~K}$. Here, the temperature of the droplet, which is relatively low around the melting point temperature, was controlled by blowing $\mathrm{He}$ gas on the droplet surface. Because the density of molten iron is relatively large, for instance, in comparison with that of molten silicon, a large electromagnetic force is required to levitate the droplet, and consequently, the heat generation rate in the droplet also increases, resulting in the droplet being excessively heated.

In the experiment, the phase lag between the modulated light and the temperature variations detected by the pyrometer, $\Delta \phi_{\mathrm{s}}$, was measured for modulated light of various frequencies, $\omega$. Then, the experimentally obtained relationship between $\Delta \phi_{\mathrm{s}}$ and $\omega$ was fitted by a mathematical model to estimate the thermal conductivity, where the unsteady-state heat conduction equation for the droplet with radiative heat transfer to the ambient was simplified and transformed to steady-state linear equations (Tsukada et al., 2007).

In this work, the effect of a static magnetic field on the thermal conductivity of molten nickel was also investigated using an electromagnetic levitator, where the geometry of RF coils and the atmosphere condition were the same as those for molten iron shown in Figure 1. The purity and mass of the nickel sample used here were $99.5 \%$ and $1.5 \mathrm{~g}$, respectively. For molten nickel, the spot radius of the pyrometer was $1.5 \mathrm{~mm}$, the $e^{-2}$ radius of the laser beam was $2.0 \mathrm{~mm}$, and the amplitude of the laser power was 9.2 or $13.8 \mathrm{~W}$. The static magnetic field applied to the molten nickel droplet was between 4 and $10 \mathrm{~T}$, and the measurement temperature range was between 1520 and $1980 \mathrm{~K}$.

\section{Results and Discussion}

Figure 2 shows the numerical results-velocity profiles and isotherms - for a molten iron droplet with a diameter of $7 \mathrm{~mm}$; the applied static magnetic fields $B_{0}$ in Figure 1 are (a) $4 \mathrm{~T}$ and (b) $10 \mathrm{~T}$, respectively. In both cases, the solutions reached a steady state. From the figures, it is found that convection in the droplet is markedly suppressed upon applying a static magnetic field and increasing the magnetic field, similar to the case of a molten silicon droplet (Sugioka et al., 2010). In addition, upon comparing the temperature distributions of the droplets subjected to static magnetic fields of 4 and $10 \mathrm{~T}$, it can be seen that the temperature field is strongly affected by convection when the applied magnetic field is relatively small. This means that the measured thermal conductivity is also affected by convection in the case of relatively small static magnetic fields.

Figure 3 shows the calculated temperature responses at the lower part of the molten iron droplet surface when the upper part of the droplet is irradiated with a modulated laser beam with a frequency of $0.1 \mathrm{~Hz}$ and a static magnetic field of $10 \mathrm{~T}$ is applied. It was found that the temperature increases in an oscillatory manner and
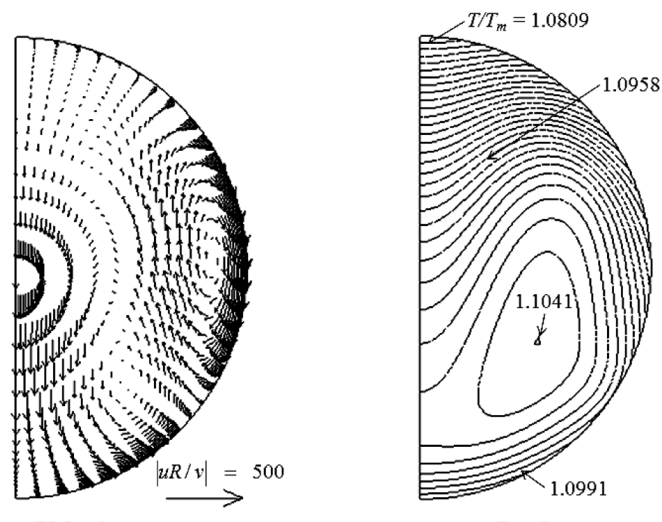

(a) $4 \mathrm{~T}$

Isotherms

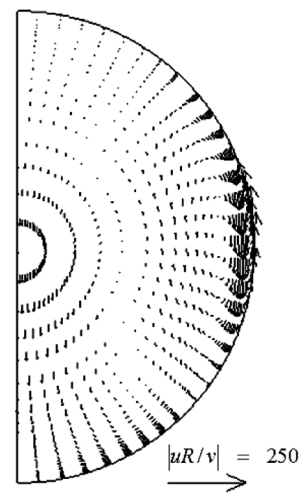

Velocity vectors

(b) $10 \mathrm{~T}$

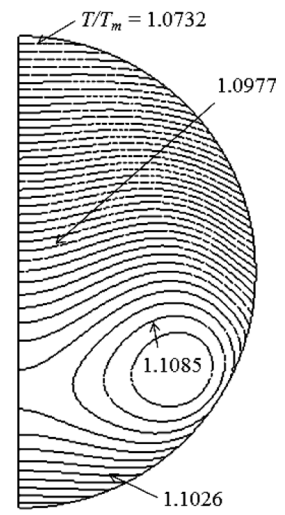

Isotherms

Fig. 2 Effect of the static magnetic field on the velocity and temperature fields in a molten iron droplet

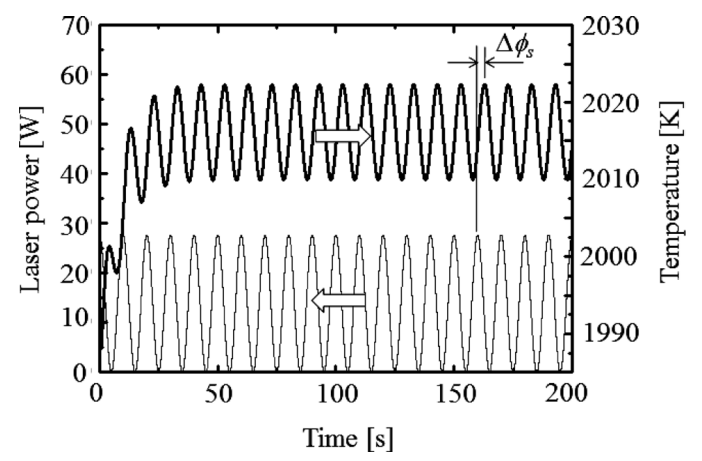

Fig. 3 Calculated temperature variations at the lower part of the molten iron droplet under a static magnetic field of $10 \mathrm{~T}$

ultimately reaches the stationary modulation state, which is characterized by a constant average temperature and amplitude. In the figure, it can be seen that there is a phase lag $\Delta \phi_{\mathrm{s}}$ between the power of the modulated light and the temperature variations.

Numerical simulations similar to those associated with Figure 3 were carried out by varying the frequency of the modulated laser beam, $\omega$, and the static magnetic 


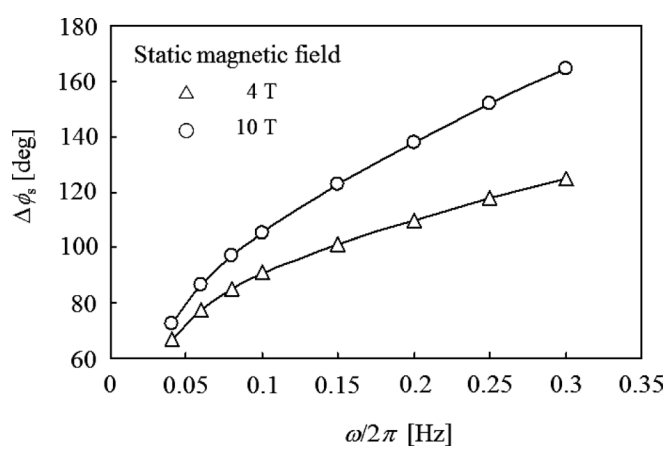

Fig. 4 Relation between $\omega$ and $\Delta \phi_{\mathrm{s}}$ for molten iron

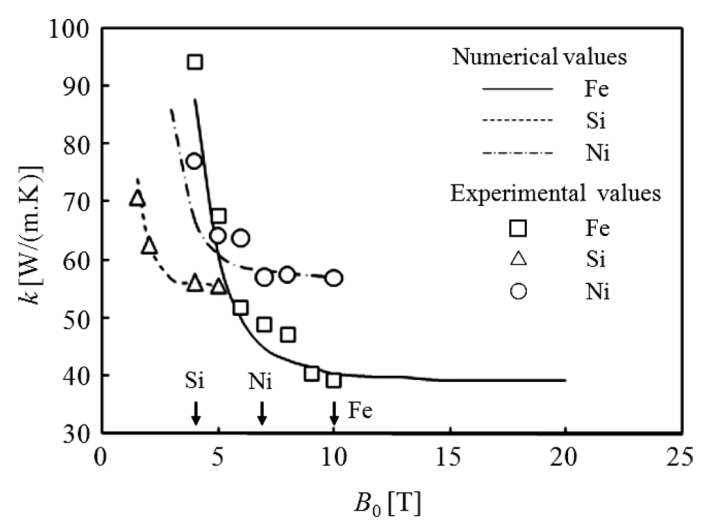

Fig. 5 Effect of the static magnetic field on the thermal conductivity

field, $B_{0}$, and the relationship between $\Delta \phi_{\mathrm{s}}$ and $\omega$ was obtained for a variety of static magnetic fields. The plots in Figure 4 show the relationships between $\Delta \phi_{\mathrm{s}}$ and $\omega$ for $B_{0}=4$ and $10 \mathrm{~T}$. Then, the mathematical model proposed in our previous work (Tsukada et al., 2007) was adopted to obtain the thermal conductivity from the plots in Figure 4, as shown by the fitted lines in the figure. Consequently, the thermal conductivity for each static magnetic field was obtained.

Figure 5 shows the effect of $B_{0}$ on the thermal conductivity $k$ of molten iron; the effect is obtained numerically from the relationships between $\Delta \phi_{\mathrm{s}}$ and $\omega$ shown in Figure 4. Figure 5 also shows the measured thermal conductivities of molten iron, those of molten nickel obtained numerically and experimentally in this work, and those of molten silicon obtained in a previous work (Sugioka et al., 2010). Each experimental value plotted in the figure is the average value of thermal conductivities measured in a specific temperature range for a fixed static magnetic field; the averaging of measurements made in a range of temperatures is valid because the temperature dependence of thermal conductivity is very small for molten iron and nickel (Sugie et al., 2010). From the figure, it can be observed that the numerical results are in good agreement with the experimental ones. The thermal conductivity increases suddenly; that is, it is

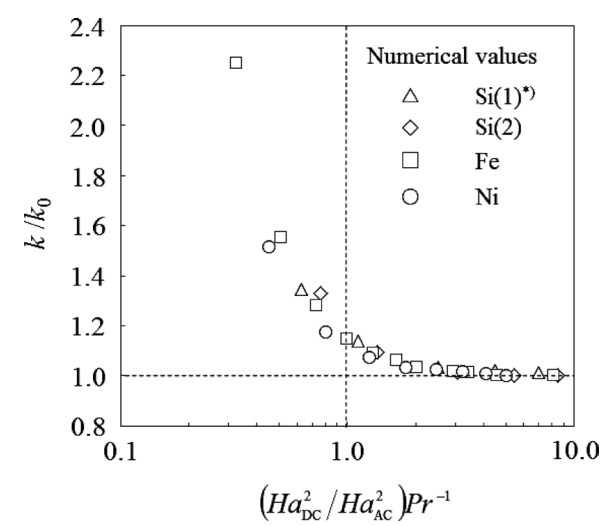

Fig. 6 Correlation between thermal conductivity and static magnetic field. $\left.{ }^{*}\right) \mathrm{Si}(1)$ represents the results of Sugioka et al. (2010)

strongly affected by the convection in the droplet (shown in Figure 2) when the static magnetic field is relatively small. In contrast, the thermal conductivity reaches the value corresponding to the absence of convection as the static magnetic field increases. These results suggest that the magnetic field should be more than $10 \mathrm{~T}$ for molten iron, more than $7 \mathrm{~T}$ for molten nickel, and more than $4 \mathrm{~T}$ for molten silicon so that the real thermal conductivity (without any convective contribution) of each molten material can be measured by the EML technique.

In Figure 5, it is observed that the dependence of the measured thermal conductivity on the static magnetic field strength is different in the three molten materials. This is because of differences in the thermophysical properties, the geometry of the RF coils, and electric current in the RF coils. In this work, the effect of such factors on the value of the thermal conductivity measured by the EML technique was investigated using nondimensional parameters, i.e., $k / k_{0}$ and $\left(H a_{\mathrm{DC}}^{2} / \mathrm{Ha}_{\mathrm{AC}}^{2}\right) \mathrm{Pr}^{-1}$, as shown in Figure 6. In the figure, $k / k_{0}$ is the ratio of apparent thermal conductivity, which includes the contributions of both conduction and melt convection, to the real thermal conductivity, and only the values corresponding to the numerical results in Figure 5 are shown because the numerical results are in good agreement with the experimental ones. Although both $\mathrm{Si}(1)$ and $\mathrm{Si}(2)$ are the numerical results for molten silicon, $\mathrm{Si}(1)$ represents the results obtained in a previous work (Sugioka et al., 2010) and $\mathrm{Si}(2)$ represents those calculated in this work, where the radius of a droplet and RF coil geometry for $\mathrm{Si}(2)$ are the same as those for molten Fe but are different from those for $\mathrm{Si}(1) . P r$ is the Prandtl number, defined as $C_{p} \mu / k_{0} . H a_{\mathrm{DC}}$ is the Hartmann number based on the static magnetic field, $H a_{\mathrm{AC}}$ is the Hartmann number based on the alternating magnetic field generated by RF coils, and the ratio $\left(\mathrm{Ha}_{\mathrm{DC}}^{2} / \mathrm{Ha}_{\mathrm{AC}}^{2}\right)$ represents the ratio of the electromagnetic force generated for suppressing melt convection to that generated for driving melt convection in an electromagnetically levitated droplet. However, 
since the electromagnetic force generated by the alternating magnetic field is affected by both the electric current in the RF coils and coil geometry, it is not easy to estimate the order of magnitude of the force. In the experiment and numerical simulation, the electric current in the $\mathrm{RF}$ coil was determined so that the molten droplet could be stably levitated at a given position relative to the RF coil with a given geometry. Since the levitation of the droplet depends on the balance between the electromagnetic force and gravity force acting on the droplet, the order of magnitude of the electromagnetic force required for driving melt convection in the droplet was estimated by considering the gravity force. Therefore, the value of $\left(H a_{\mathrm{DC}}^{2} / H a_{\mathrm{AC}}^{2}\right)$ in Figure 6 was evaluated by considering a nondimensional parameter, $\sigma B_{0}^{2} \mu / R \rho^{2} g$. In the figure, it is observed that melt convection could be suppressed sufficiently at $\left(\mathrm{Ha}_{\mathrm{DC}}^{2} / \mathrm{Ha}_{\mathrm{AC}}^{2}\right) \mathrm{Pr}^{-1}>4.0$.

\section{Conclusions}

In order to quantitatively investigate the effect of convection in electromagnetically levitated molten droplets of iron and nickel on the thermal conductivities of the droplets measured by the electromagnetic levitation (EML) technique in the presence of a static magnetic field, numerical simulations for melt convection in the droplet and for the measurement of thermal conductivity based on the periodic laser heating method were carried out. By comparing the measured thermal conductivities of the molten materials with those obtained numerically, it was found that the numerical simulations could sufficiently explain the measurement of thermal conductivity by the EML technique in a static magnetic field. It is suggested that magnetic fields of 10 and $7 \mathrm{~T}$ are sufficient to measure the real thermal conductivities of molten iron and nickel by the EML technique, respectively. Moreover, the correlation between the static magnetic field and the contribution of melt convection to the measured thermal conductivity was investigated using a nondimensional parameter - the ratio of the electromagnetic force generated by the static magnetic field to that generated by the alternating current in the RF coils.

\section{Acknowledgement}

This development was supported by SENTAN, JST.

\begin{tabular}{|c|c|c|}
\hline \multicolumn{3}{|c|}{ Nomenclature } \\
\hline$B_{0}$ & $=$ magnetic flux density & {$[\mathrm{T}]$} \\
\hline$C_{\mathrm{p}}$ & $=$ specific heat & {$[\mathrm{J} / \mathrm{kg} \cdot \mathrm{K}]$} \\
\hline$g$ & $=$ gravitational acceleration & {$\left[\mathrm{m} / \mathrm{s}^{2}\right]$} \\
\hline $\mathrm{Ha}$ & $=$ Hartmann number & [-] \\
\hline$k$ & $=$ apparent thermal conductivity & {$[\mathrm{W} / \mathrm{m} \cdot \mathrm{K}]$} \\
\hline$k_{0}$ & $=$ real thermal conductivity & {$[\mathrm{W} / \mathrm{m} \cdot \mathrm{K}]$} \\
\hline $\operatorname{Pr}$ & $=$ Prandtl number & {$[-]$} \\
\hline$R$ & $=$ radius of droplet & {$[\mathrm{m}]$} \\
\hline$t$ & $=$ time & \\
\hline$\Delta \phi_{\mathrm{s}}$ & $\begin{array}{l}=\text { phase lag between modulated } \\
\text { sponse of temperature }\end{array}$ & er and \\
\hline
\end{tabular}

$\begin{array}{rlr}\mu & =\text { viscosity } & {[\mathrm{Pa} \cdot \mathrm{s}]} \\ \rho & =\text { density } & {\left[\mathrm{kg} / \mathrm{m}^{3}\right]} \\ \sigma & =\text { electric conductivity } & {[1 / \Omega \cdot \mathrm{m}]} \\ \omega & =\text { frequency of modulated laser power } & {[\mathrm{rad} / \mathrm{s}]}\end{array}$

\section{Literature Cited}

Bojarevics, V. and K. Pericleous; "Modelling Electromagnetically Levitated Liquid Droplet Oscillations," ISIJ Int., 43, 890-898 (2003)

Egry, I., A. Diefenbach, W. Dreier and J. Piller; "Containerless Processing in Space-Thermophysical Property Measurements Using Electromagnetic Levitation," Int. J. Thermophys., 22, 569-578 (2001)

Fukuyama, H., K. Takahashi, S. Sakashita, H. Kobatake, T. Tsukada and S. Awaji; "Noncontact Modulated Laser Calorimetry for Liquid Austenitic Stainless Steel in DC Magnetic Field," ISIJ Int., 49, 1436-1442 (2009)

Gale, W. F. and T. C. Totemeier; Smithells Metals Reference Book, 8th ed., Elsevier, Amsterdam, the Netherland (2004)

Hyers, R. W.; "Fluid Flow Effects in Levitated Droplets," Meas. Sci. Technol., 16, 394-401 (2005)

Kobatake, H.; personal communication (2010)

Kobatake, H., H. Fukuyama, I. Minato, T. Tsukada and S. Awaji; "Noncontact Modulated Laser Calorimetry of Liquid Silicon in a Static Magnetic Field," Appl. Phys. Lett., 90, 094102 (2007)

Kobatake, H., H. Fukuyama, I. Minato, T. Tsukada and S. Awaji; "Noncontact Measurement of Thermal Conductivity of Liquid Silicon in a Static Magnetic Field," J. Appl. Phys., 104, 054901 (2008)

Kobatake, H., H. Fukuyama, T. Tsukada and S. Awaji; "Noncontact Modulated Laser Calorimetry in a DC Magnetic Field for Stable and Supercooled Liquid Silicon," Meas. Sci. Technol., 21, 025901 (2010a).

Kobatake, H., H. Khosroabadi and H. Fukuyama; "Noncontact Measurement of Normal Spectral Emissivity, Heat Capacity and Thermal Conductivity of Liquid Ni in a DC Magnetic Field," Proc. of eTherm 2010, pp. 122-124, Tsukuba, Japan (2010b)

Li, B. Q. and S. P. Song; "Thermal and Fluid Flow Aspects of Electromagnetic and Electrostatic Levitation-A Comparative Modeling Study," Microgravity Sci. Technol., XI, 134-143 (1998)

Mills, K. C., B. J. Monaghan and B. J. Keene; "Thermal Conductivities of Molten Metals: Part1 Pure Metals," Int. Mater. Rev., 41, 209-242 (1996)

Nishi, T., H. Shibata, H. Ohta and Y. Waseda; "Thermal Conductivities of Molten Iron, Cobalt, and Nickel by Laser Flash Method," Metall. Mater. Trans. A, 34, 2801-2807 (2003)

Nishizuka, T., Y. Sato, T. Takamizawa, K. Sugisawa and T. Yamamura; "Density and Viscosity of Molten Fe, Ni and Co," Proc. of 16th European Conf. on Thermophysical Properties, CD-ROM, London, U.K. (2002).

Ostrovskii, O. I., V. A. Ermachenko, V. M. Popov, V. A. Grigoryan and L. E. Kogtan; "Thermophysical Properties of Liquid Iron, Cobalt, and Nickel,” Russ. J. Phys. Chem., 54, 739-741 (1980)

Sugie, K., H. Kobatake, H. Fukuyama, Y. Baba, K.-I. Sugioka and T. Tsukada; "Development of Thermophysical Property Measurement for Liquid Fe Using Noncontact Laser Modulation Calorimetry," Tetsu-to-Hagane, 96, 673-682 (2010)

Sugioka, K.-I., T. Tsukada, H. Fukuyama, H. Kobatake and S. Awaji; "Effect of Static Magnetic Field on Thermal Conductivity Measurement of a Molten Si Droplet by an EML Technique: Comparison between Numerical and Experimental Results," Int. J. Heat Mass Transfer, 53, 4228-4232 (2010)

Touloukian, Y. S., R. W. Powell, C. Y. Ho and P. G. Klements; The TPRC Data Series, vol. 1, p. 169, Plenum, New York, U.S.A. (1970)

Tsukada, T., H. Fukuyama and H. Kobatake; "Determination of Thermal Conductivity and Emissivity of Electromagnetically Levitated High-Temperature Droplet Based on the Periodic Laser-Heating Method: Theory," Int. J. Heat Mass Transfer, 50, 3054-3061 
(2007)

Tsukada, T., K.-I. Sugioka, T. Tsutsumino, H. Fukuyama and H. Kobatake; "Effect of Static Magnetic Field on a Thermal Conductivity Measurement of a Molten Droplet Using an Electromagnetic Levitation Technique," Int. J. Heat Mass Transfer, 52 5152-5157 (2009)

Zinovyev, V. Y., V. F. Polev, S. G. Taluts, G. P. Zinovyeya and S. A
Ilinykh; "Diffusivity and Thermal Conductivity of 3d-Transition Metals in Solid and Liquid States," Phys. Met. Metall., 61, 85-92 (1986)

Zong, J. H., B. Li and J. Szekely; "The Electrodynamic and Hydrodynamic Phenomena in Magnetically-Levitated Molten Droplets: 1. Steady-State Behavior," Acta Astronaut., 26, 435-449 (1992) 